\title{
Immune recovery uveitis in HIV patients with cytomegalovirus retinitis in the era of HAART therapy-a 5-year study from Singapore
}

\author{
Tun Hang Yeo ${ }^{1 *}$ (D), Tun Kuan Yeo ${ }^{1}$, Elizabeth P. Wong ${ }^{1}$, Rupesh Agrawal ${ }^{1}$ and Stephen C. Teoh ${ }^{1,2}$
}

\begin{abstract}
Background: The aim of this study is to analyse the clinical features of HIV patients with cytomegalovirus retinitis (CMVR) developing immune recovery uveitis (IRU) while on highly active antiretroviral therapy (HAART) and to identify the risk factors, visual outcomes and complications of IRU.

Results: Majority $(n=26,86.7 \%)$ of patients were male, with $76.7 \%(n=23)$ of patients having bilateral disease. Twenty-seven eyes (50.9\%) had both anterior uveitis and vitritis. The median CD4 at IRU was $210 \mathrm{cells} / \mathrm{\mu L}$ (IQR 140-279), with $86.7 \%$ having CD4 $>100$ cells/ $/ \mathrm{L}$. The median duration from initiation of HAART to IRU was significantly different between those $<50$ years old (median 763 days, IQR 174-1872 days) and those $\geq 50$ years old (median 161 days, IQR 84.5-278 days). Fourteen eyes (26.4 \%) had loss of one or more Snellen lines visual acuity at 6 months while the rest maintained or improved vision. Complications developed in 21 eyes, with cataract (66.7 \%), glaucoma and ocular hypertension (33.3\%) being the most common. The risk of complications was associated with the absolute difference in CD4 counts at IRU and at HAART commencement $(p=0.041)$. Age was also negatively associated with the duration from HAART to IRU ( $p=0.005$, Spearman's rho coefficient $=-0.503)$.
\end{abstract}

Conclusions: It is common to have both anterior uveitis and vitritis in IRU. There was a positive association between the increase in CD4 from HIV to IRU diagnoses and the risk of developing complications. Younger patients appeared to develop IRU later than older patients after HAART, suggesting that long-term follow-ups are essential for these patients.

Keywords: Immune recovery uveitis, Human immunodeficiency virus, Cytomegalovirus retinitis, Highly active antiretroviral therapy, Immune reconstitution inflammatory syndrome

\section{Background}

Immune reconstitution inflammatory syndrome (IRIS) is a group of disorders that has been described in HIV patients who are on highly active antiretroviral therapy (HAART). This phenomenon is associated with a paradoxical or exaggerated reactivation of the immune system in the background of a wide range of pathologies such as Kaposi's sarcoma, tuberculosis, cryptococcal infection and cytomegalovirus [1].

\footnotetext{
* Correspondence: tunhang.yeo@gmail.com

'National Healthcare Group Eye Institute, Tan Tock Seng Hospital, Singapore

308433, Singapore

Full list of author information is available at the end of the article
}

Immune recovery uveitis (IRU) is one manifestation where immune-deficient patients with prior cytomegalovirus retinitis (CMVR) experience an increase in intraocular inflammation following HAART. It is presumed to be mediated by the recovery of immune responses specific to residual cytomegalovirus (CMV) antigens in the eye [2]. IRU is an important cause of visual morbidity in HIV-infected patients with CMVR in the era of HAART [3]. Although immune recovery associated with HAART has allowed some patients to discontinue specific antiCMV therapy, the rejuvenated immune response can be 
associated with chronic sight-threatening inflammation. Visual complications of IRU include cataract, cystoid macular edema (CME), glaucoma and epiretinal membrane formation [4-10]. Reported risk factors include a low CD4 count at the time of initiation of HAART and larger areas of CMVR [3]. With the rising prevalence of HIV patients and greater accessibility and availability of HAART worldwide, IRU is an increasingly important disease entity. We aim to analyse the characteristics of IRU in HIV patients on HAART in Singapore, to identify risk factors for the development of IRU and to determine their visual outcomes.

\section{Methods}

A retrospective analysis of clinical records of 30 consecutive IRU patients presenting to the Eye Clinic at the Communicable Disease Centre (CDC), Singapore, between March 2004 and September 2009 was performed. Ethics approval was obtained from the local ethics review board in accordance with the Declaration of Helsinki.

The CDC is the main site of referral and management for HIV in Singapore. IRU was diagnosed when there is an increase in any form of intraocular inflammation from an inactive state of CMV retinitis secondary to immune recovery after initiation of HAART, as evidenced by an increase in CD4 count by 50 or more cells $/ \mu \mathrm{L}$ from the nadir CD4 count to a level of more than 100 cells $/ \mu \mathrm{L}$ [3]. Despite a CD4 count of less than 100 cells $/ \mu \mathrm{L}$ at diagnosis, a small subset of patients are also deemed to have IRU. These patients would have an increase in intraocular inflammation, which is correlated with a rise in CD4 count that could not be clinically attributed to a reactivation of CMVR.

All of the patients had prior CMV retinitis treated with an intravitreal ganciclovir regimen, as previously described [11]. Two patients were also treated with intravenous ganciclovir, and one received oral valganciclovir. Patients with concomitant infective or other autoimmune causes of inflammation were excluded from this study. All patients underwent Snellen bestcorrected visual acuity (BCVA) assessment, tonometry and dilated slit-lamp biomicroscopy and indirect ophthalmoscopy examinations. Clinical data obtained included patient demographics (age, gender and ethnicity); duration between HIV, CMVR and IRU diagnoses; BCVA at presentation and at 6 months; corresponding CD4 counts and CD4/CD8 ratios; duration from initiation of HAART to IRU development; presenting signs and symptoms; CMVR zone and area of involvement; IRU severity and location; and treatment regimes. The location of CMVR lesion in each eye was categorised into three zones. Zone 1 was defined as the area within $1500 \mu \mathrm{m}$ of the optic nerve or within $3000 \mu \mathrm{m}$ of the fovea. Zone 2 extended from the borders of zone 1 to the vortex veins, and zone 3 involves the retina peripheral to zone 2 [12]. For patients with CMVR involving multiple zones, the zone nearest to the macula was reported.

Descriptive analysis was performed for demographics, visual acuity, complications and IRU and CMVR characteristics, with categorical variables summarised with frequencies and percentages and continuous variables summarised as either means with standard deviations or medians with ranges. Spearman's rho was calculated to check for association between CD4 counts and durations. Independent sample $t$ test and Mann-Whitney $U$ test were used for comparison of age, durations and CD4 counts between patient groups with and without complications, respectively. Logistic regression was used to understand the relationship between variables. A $p$ value less than 0.05 was considered to indicate statistical significance. Data analysis was carried out using IBM SPSS Statistics (version 19, IBM Corp., NY, USA).

\section{Results \\ IRU characteristics}

Twenty-six patients (86.7\%) were male, and the median age at IRU diagnosis was 49.5 years old (interquartile range (IQR) 41-56 years). Chinese patients $(n=25)$ formed the majority, corresponding to the ethnic distribution of the local population [13]. Only eyes $(n=53)$ with previous CMVR, in this cohort, developed IRU. Twenty-three patients $(76.7 \%)$ presented with bilateral IRU. Twenty-seven IRU eyes (50.9\%) presented with both anterior uveitis and vitritis. Twenty-two IRU eyes $(41.5 \%)$ presented with anterior uveitis alone, while one eye $(1.9 \%)$ presented with only vitritis. The median CD4 count at HIV diagnosis of these patients was 20 cells $/ \mu \mathrm{L}$ (IQR 15.0-62.0), while the median CD4 count at IRU diagnosis was 210 cells/ $\mu \mathrm{L}$ (IQR 140-279), with $86.7 \%$ having CD4 $>100$ cells $/ \mu \mathrm{L}$. Two of the four patients with CD4 count less than 100 cells/ $\mu \mathrm{L}$ had more than 50 cells $/ \mu \mathrm{L}$ increase in CD4 count at IRU diagnosis. The median increase in CD4 at IRU was 155.5 cells $/ \mu \mathrm{L}$ (IQR 66.0-228.0). The median duration from HIV diagnosis to initiation of HAART was 100.5 days (IQR 61.0-210.0), while the median duration from initiation of HAART to IRU was 214 days (IQR 118.0-763.0). Thirteen IRU patients (43.3\%) were asymptomatic at presentation. Of the remaining 17 IRU patients, the most common symptom was blurring of vision alone $(n=11,36.7 \%)$ followed by floaters $(n=4,13.3 \%)$. All patients were treated with topical steroids, with eight patients requiring additional sub-tenon steroid injections and two patients receiving oral steroids (Tables 1 and 2). 
Table 1 IRU characteristics

\begin{tabular}{ll}
\hline Number of patients, $n$ & 30 \\
Gender, $n(\%)$ & $26(86.7)$ \\
Male & $4(13.3)$ \\
Female & \\
Race, $n(\%)$ & $25(83.3)$ \\
Chinese & $3(10.0)$ \\
Malay & $1(3.3)$ \\
Indian & $1(3.3)$ \\
Others & \\
Age, years; mean (SD) & $47.9(10.7)$ \\
At IRU diagnosis & \\
CD4 count, cells/ $\mu$ L; median (IQR) & $20.0(15.0-62.0)$ \\
At HAART commencement & $210.0(139.8-289.5)$ \\
At IRU diagnosis & $28.5(10.5-61.8)$ \\
At CMVR diagnosis & $155.5(66.0-228.0)$ \\
Difference from HAART to IRU diagnosis & \\
Duration, days; median (IQR) & 100.5 (61.0-210.0) \\
From HIV diagnosis to HAART commencement & $214.0(118.0-763.0)$ \\
From HAART initiation to IRU diagnosis & $136.5(26.0-618.5)$ \\
From CMVR diagnosis to IRU diagnosis & \\
\hline
\end{tabular}

\section{CMVR characteristics}

All of the patients had CMVR in at least one eye. Fiftythree of the 60 eyes (88.3\%) had previous CMVR. The median duration from HIV diagnosis to CMVR was 283.0 days (IQR 142.0-539.5), while the median duration from CMVR diagnosis to anti-CMVR treatment was 0 days (range -2 to 16 ), with 24 patients receiving treatment on the same day as CMVR diagnosis. The median CD4 count at CMVR diagnosis in our patients

Table 2 IRU-presenting characteristics

\begin{tabular}{ll}
\hline Number of patients, $n$ & 30 \\
Symptoms, $n$ (\%) & $11(36.7)$ \\
Blurring of vision & $4(13.3)$ \\
Floaters & $1(3.3)$ \\
Pain & $1(3.3)$ \\
Blurring of vision and floaters & $13(43.3)$ \\
None & 53 \\
Number of IRU eyes, $n$ & \\
Presenting features, $n$ (\%) & $22(41.5)$ \\
Anterior uveitis alone & $1(1.9)$ \\
Vitritis alone & $27(50.9)$ \\
Anterior uveitis and vitritis & $2(3.8)$ \\
Anterior uveitis and vitreous haemorrhage & $1(1.9)$ \\
\hline
\end{tabular}

was 28.5 cells $/ \mu \mathrm{L}$ (IQR $11.0-60.5)$. Most eyes $(n=23$, $43.4 \%$ ) had previous CMVR involvement in zone 2 . This was followed by zone 1 CMVR involvement in 15 eyes (28.3\%) and zone 3 involvement in 11 eyes (20.8\%). The majority of eyes $(n=40,75.5 \%)$ had $<25 \%$ area of involvement, while nine eyes $(17.0 \%)$ had $25-50 \%$ area of involvement and four eyes $(7.5 \%)$ had $>50 \%$ area of involvement (Table 3).

\section{Visual outcome}

The BCVA of IRU patients was assessed at diagnosis of IRU and at 6 months. The range of visual acuity at presentation was 6/4 to NPL, with 19 eyes (35.8\%) having BCVA worse than 6/12. At 6 months, 18 eyes (34\%) had BCVA worse than 6/12. Six (11.3\%) and two eyes (3.8\%) had a loss of one and two Snellen lines, respectively. Six eyes (11.3\%) had a loss of three Snellen lines or more. These were due to glaucoma, cataract or persistent inflammation. The remaining patients had visual acuities that were maintained or better than at IRU presentation (Table 4).

\section{Complications}

Twenty-one eyes (39.6\%) developed complications. The most common complication was cataract $(n=14,66.7 \%)$, followed by glaucoma and ocular hypertension $(n=7$, $33.3 \%)$. There were no cases of CME during the course of this study. Mann-Whitney $U$ test showed an association between the presence of complications and the absolute difference in CD4 counts at IRU and at HAART commencement $(p=0.041)$. For every 50 cells $/ \mu \mathrm{L}$ increase in CD4 at IRU diagnosis, patients were 1.28 times more likely to develop complications $(p=0.084)$. Although not statistically significant, the higher CD4 count at IRU diagnosis also appeared to be associated with the development of complications $(p=0.057)$. CMVR characteristics (zone and area of involvement) were not found to be associated with

Table 3 CMV characteristics

\begin{tabular}{ll}
\hline Number of eyes, $n$ & 53 \\
Location, $n(\%)$ & $15(28.3)$ \\
Zone 1 & $23(43.4)$ \\
Zone 2 & $11(20.8)$ \\
Zone 3 & \\
CMVR involvement retinal area, $n$ (\%) & $40(75.5)$ \\
$<25 \%$ & $9(17.0)$ \\
$25-50 \%$ & $4(7.5)$ \\
$>50 \%$ & \\
Duration, days; median (IQR) & $283.0(142.0-539.5)$ \\
From HIV diagnosis to CMVR diagnosis & $0.0(0.0-0.0)$ \\
From CMVR diagnosis to anti-CMVR treatment & \\
\hline
\end{tabular}


Table 4 Visual outcome

\begin{tabular}{lc}
\hline Number of affected eyes, $n$ & 53 \\
Range of VA at IRU presentation, $n(\%)$ & $34(64.2 \%)$ \\
$6 / 6$ to $6 / 12$ & $12(22.6 \%)$ \\
$6 / 15$ to $6 / 45$ & $7(13.2 \%)$ \\
$6 / 60$ or worse & $35(66.0 \%)$ \\
Range of VA at 6 -month follow-up post IRU diagnosis, $n(\%)$ \\
$6 / 6$ to $6 / 12$ & $10(18.9 \%)$ \\
$6 / 15$ to $6 / 45$ & $8(15.1 \%)$ \\
$6 / 60$ or worse & $6(11.3 \%)$ \\
VA change from IRU diagnosis to 6-month follow-up, $n(\%)$ \\
Worsen by at least 3 lines & $2(3.8 \%)$ \\
Worsen by 2 lines & $6(11.3 \%)$ \\
Worsen by 1 line & $18(34.0 \%)$ \\
No change & $7(13.2 \%)$ \\
Improved by 1 line & $6(11.3 \%)$ \\
Improved by 2 lines & $8(15.1 \%)$ \\
Improved by at least 3 lines &
\end{tabular}

the development of complications $(p=0.534$ and $p=0.715$, respectively).

\section{Risk factors}

Age was found to be negatively associated with the duration taken to develop IRU after initiation of HAART $(p=0.005$, Spearman's rho coefficient $=-0.503)$. Median duration from HAART initiation to development of IRU was 763 days (IQR 174-1872 days) and 161 days (IQR 84.5-278 days) for patients $<50$ years old and patients $\geq 50$ years old, respectively. The CD4 counts at HIV diagnosis, at initiation of HAART and at IRU diagnosis were not found to be significantly associated with the duration from HAART to IRU diagnosis. Both the absolute and the percentage increase in CD4 counts were also not significantly associated with the duration taken to develop IRU after initiation of HAART. CMVR zone or area of involvement was not found to be associated with the duration of IRU development from initiation of HAART.

\section{Discussion}

HIV-related mortality has decreased dramatically in the era of HAART. However, it is now evident that the commencement of HAART can also potentially lead to considerable morbidity and mortality in the form of IRIS, especially in the first 6 months [14]. IRU is an important presentation in this broad disease group which also includes tuberculosis-associated IRIS and Kaposi's sarcoma IRIS [1]. This is the first paper discussing the characteristics of IRU and its complications in Singapore and Southeast Asia. As the Communicable Disease Centre in Singapore manages and treats the majority of local HIV patients [15], our patient cohort is highly reflective of IRU diagnosed locally. The number of HIV-positive patients in Singapore has been gradually increasing, with 454 newly diagnosed cases in 2013 and a total of 6229 patients diagnosed since 1985 [16]. The number of patients with CMVR has increased correspondingly, with 224 patients diagnosed at the CDC from 2005 to 2010 [17]. IRU is most commonly associated with previous CMVR in patients who have experienced immune recovery.

Similar to previous studies, vitritis is the most common presentation in our cohort $[5,10,18,19]$. There are also a significant proportion of patients who had anterior uveitis. Although anterior uveitis is commonly reported $[5,9,10,20]$, most studies have focused their diagnosis of IRU on the presence of vitritis and associated CME [4, 6, 9, 18, 19].

Patients with larger areas of CMVR involvement were reported to have higher risk of IRU development [9]. In contrast, the majority of eyes in our cohort had $<25 \%$ area of previous CMVR involvement. Arevalo et al. did not find an association between CMVR area and risk of IRU development [21]. This suggests that small CMVR lesions are common and eyes without large areas of CMVR invovement are similarly at risk of developing IRU.

Within our cohort, we did not find any association between CD4 counts at HIV diagnosis, at initiation of HAART or at IRU diagnosis, with the development of IRU. However, the duration from the initiation of HAART to IRU was found to be longer in younger patients ( $<50$ years old). Hartigan-O'Connor et al. previously reported that IRU is most likely to develop in patients with the greatest degree of immune dysfunction prior to HAART [22]. Additionally, older age groups have been correlated with quicker seroconversion to AIDS in the pre-HAART era, as well as higher rates of immunologic failure and persistent HIV viraemia in the HAART era [23]. Thus, it is plausible that younger HIV patients have a more intact immune system that may not have such heightened sensitivity to CD4 $\mathrm{T}$ cell responses, consequently delaying their development of IRU until the occurrence of further immune dysfunction.

The most common complications of IRU in our cohort were cataract formation, glaucoma and ocular hypertension. Our findings differ from other reports where CME and epiretinal membrane were the most common complications. For instance, Robinson et al. reported that of the IRU eyes in their cohort, $91 \%$ developed CME, $52 \%$ developed cataract and $30 \%$ developed epiretinal membrane [5]. Cataract, CME and epiretinal membrane typically caused moderate visual loss $[3,5,7]$.

No cases of CME were reported during the course of this study. Although CME is often described and is a serious sight-threatening complication of IRU, we believe 
that it is not a common feature, especially if the inflammation is treated earlier. This is particularly pertinent as there is currently no consensus on the ideal time to start treatment of IRU. In fact, the majority of studies have suggested that mild and asymptomatic ocular inflammation usually resolves without treatment; treatment is instituted in more severe inflammation and in patients who become symptomatic $[6,8]$.

IRU has been described as a transient form of inflammation [19]. Still, others report that the persistence of inflammation in IRU is the cause of complications like CME and epiretinal membrane [6, 18]. Based on our findings, we propose the early treatment of ocular inflammation in IRU patients to prevent sight-threatening complications of CME and epiretinal membrane. Nonetheless, the judicious use of topical steroids in treating asymptomatic patients must be balanced against the possible complications of steroid use.

The risk of IRU complications was found to be higher with greater CD4 count increase from HIV to IRU diagnosis. The greater inflammatory response mounted in these immune reconstituted patients, together with a robust increase in the number of viable $\mathrm{T}$ lymphocytes, results in severe and chronic inflammation [24]. The increased inflammatory response can lead to cataract formation, raised intraocular pressure and glaucoma. Conversely, the development of cataract and raised intraocular pressure may also be the result of aggressive steroid therapy, which is often instituted in these patients. However, the mode of steroid therapy did not appear to be associated with the risk of development of complications in this study.

The presence of IRU at baseline was reported to be associated with an increased risk of moderate vision loss to 6/15 [3]. The visual outcome of our IRU patient cohort was, however, fair with only eight eyes losing two or more lines of Snellen VA. $73.6 \%$ of eyes $(n=39)$ had either maintained or improved in vision. The importance of early diagnosis with aggressive and appropriate treatment can improve and preserve vision. Moreover, a number of patients were asymptomatic and were found to have IRU only on routine follow-up examinations. All HIV patients with treated CMVR should continue to have regular reviews at the eye clinic at regular intervals, even if they have high CD4 counts. Our study showed the median time from commencement of HAART to IRU development to be about 7 months. The longest time taken was 8.8 years in a 25 -year-old female. Without regular follow-up, early recognition and treatment, the visual outcomes of these patients may be less favourable.

The main limitations of this study were its retrospective design and relatively small sample size. There was also no control group. Unlike other studies, we did not routinely perform angiogram on our patients. Hence, it is possible that early CME not apparent on physical examination was missed. Nonetheless, our cohort of patients was diagnosed consecutively from the $\mathrm{CDC}$, which is reflective of the HIV patient profile in Singapore, as the CDC is the main centre for HIV treatment.

\section{Conclusions}

IRU is common and usually presents with vitritis. Early treatment of ocular inflammation can prevent the development of sight-threatening complications of IRU. The most common complication is cataract formation, and the risk of complications was positively associated with the increase in CD4 count at IRU and HIV diagnosis. Younger patients also appeared to develop IRU later than older patients after HAART, emphasising that longterm follow-ups are essential for these patients.

\section{Acknowledgements}

Nil.

\section{Authors' contributions}

THY made substantial contributions to the conception and design, analysis and interpretation of the data and the drafting of the manuscript and its critical revision for important intellectual content. TKY made substantial contributions to the conception and design, analysis and interpretation of the data and the drafting of the manuscript. EPW made substantial contributions to the analysis and interpretation of the data. RA made substantial contributions to the analysis and interpretation of the data and the critical revision of the manuscript for important intellectual content. SCT made substantial contributions to the conception and design, analysis and interpretation of the data and critical revision of the manuscript for important intellectual content. All authors read and approved the final manuscript.

\section{Competing interests}

The authors declare that they have no competing interests.

\section{Ethics approval}

Ethics approval was obtained from the local ethics review board in accordance with the Declaration of Helsinki.

\section{Author details}

${ }^{1}$ National Healthcare Group Eye Institute, Tan Tock Seng Hospital, Singapore 308433, Singapore. ${ }^{2}$ Eagle Eye Centre, Singapore, Singapore.

Received: 13 June 2016 Accepted: 27 October 2016

Published online: 07 November 2016

\section{References}

1. Müller M, Wandel S, Colebunders R et al (2010) Immune reconstitution inflammatory syndrome in patients starting antiretroviral therapy for HIV infection: a systematic review and meta-analysis. Lancet Infect Dis 10:251-61

2. Otiti-Sengeri J, Meenken C, van den Horn GJ et al (2008) Ocular immune reconstitution inflammatory syndromes. Curr Opin HIV AIDS 3:432-7

3. Thorne JE, Jabs DA, Kempen JH et al (2006) Incidence of and risk factors for visual acuity loss among patients with AIDS and cytomegalovirus retinitis in the era of highly active antiretroviral therapy. Ophthalmology 113:1432-1440

4. Nguyen QD, Kempen JH, Bolton SG, Dunn JP, Jabs DA (2000) Immune recovery uveitis in patients with AIDS and cytomegalovirus retinitis following highly active antiretroviral therapy. Am J Ophthalmol 129:634-639

5. Robinson MR, Reed G, Csaky KG et al (2000) Immune-recovery uveitis in patients with cytomegalovirus retinitis taking highly active antiretroviral therapy. Am J Ophthalmol 130:49-56 
6. Karavellas MP, Azen SP, MacDonald JC et al (2001) Immune recovery vitritis and uveitis in AIDS: clinical predictors, sequelae, and treatment outcomes. Retina 21:1-9

7. Goldberg DE, Wang H, Azen SP, Freeman WR (2003) Long term visual outcome of patients with cytomegalovirus retinitis treated with highly active antiretroviral therapy. $\mathrm{Br} J$ Ophthalmol 87:853-855

8. El-Bradey MH, Cheng L, Song M et al (2004) Long-term results of treatment of macular complications in eyes with immune recovery uveitis using a graded treatment approach. Retina 24:376-382

9. Kempen JH, Min Y, Freeman WR et al (2006) Risk of immune recovery uveitis in patients with AIDS and cytomegalovirus retinitis. Ophthalmology 113:684-694

10. Lin YC, Yang CH, Lin CP et al (2008) Cytomegalovirus retinitis and immune recovery uveitis in AIDS patients treated with highly active antiretroviral therapy in Taiwanese. Ocul Immuno Inflamm 16:83-87

11. Teoh SC, Ou X, Lim TH (2012) Intravitreal ganciclovir maintenance injection for cytomegalovirus retinitis: efficacy of a low-volume, intermediate-dose regimen. Ophthalmology 119:588-95

12. Cunningham ET Jr, Hubbard LD, Danis RP, Holland GN (2011) Proportionate topographic areas of retinal zones 1, 2, and 3 for use in describing infectious retinitis. Arch Ophthalmol 129:1507-8

13. Department of Statistics, Singapore. Population trends 2012 Available from: www.singstat.gov.sg. Accessibility verified October 20, 2015

14. Walker NF, Scriven J, Meintjes G, Wilkinson RJ (2015) Immune reconstitution inflammatory syndrome in HIV-infected patients. HIV AIDS (Auckl) 12:49-64

15. Shah JM, Leo SW, Lee TL et al (2015) A cytomegalovirus retinitis screening program: evaluation of enrollment criteria for HIV patients in Singapore. Ocul Immunol Inflamm 23:362-70

16. Ministry of Health. Update on the HIV/AIDS Situation in Singapore 2013. Available from: https://www.moh.gov.sg/content/moh_web/home/statistics/ infectiousDiseasesStatistics/HIV_Stats/update-on-the-hiv-aids-situation-insingapore-20130.html. Accessibility verified November 1, 2015

17. Teoh SC, Wang PX, Wong EPY (2012) The epidemiology and incidence of cytomegalovirus retinitis in the HIV population in Singapore over 6 years. Invest Ophthalmol Vis Sci 53:7546-7552

18. Karavellas MP, Lowder CY, Macdonald JC et al (1998) Immune recovery vitritis associated with inactive cytomegalovirus retinitis. Arch Ophthalmol 116:169-175

19. Zegans ME, Walton RC, Holland GN et al (1998) Transient vitreous inflammatory reactions associated with combination antiretroviral therapy in patients with AIDS and cytomegalovirus retinitis. Am J Ophthalmol 125:292-300

20. Henderson HWA, Mitchell SM (1998) Treatment of immune recovery vitritis with local steroids. Br J Ophthalmol 83:540-545

21. Arevalo JF, Mendoza AJ, Ferretti Y (2003) Immune recovery uveitis in AIDS patients with cytomegalovirus retinitis treated with highly active antiretroviral therapy in Venezuela. Retina 23:495-502

22. Hartigan-O'Connor DJ, Jacobson MA, Tan QX et al (2011) Development of cytomegalovirus (CMV) immune recovery uveitis is associated with Th17 cell depletion and poor systemic CMV-specific T cell responses. Clin Infect Dis 52:409-417

23. Karris MY, Kao YT, Patel D et al (2014) Predictors of virologic response in persons who start antiretroviral therapy during recent HIV infection. AIDS 28:841-849

24. Lederman M, Conici E, Landay A et al (1998) Immunologic responses associated with 12 weeks of combination antiretroviral therapy consisting of zidovudine, lamivudine, andritonavir: results of AIDS Clinical Trial Group Protocol 315. J Infect Dis 178:70-79

\section{Submit your manuscript to a SpringerOpen ${ }^{\circ}$ journal and benefit from:}

- Convenient online submission

- Rigorous peer review

Immediate publication on acceptance

- Open access: articles freely available online

- High visibility within the field

- Retaining the copyright to your article 